\section{Re-purposing Ac/Ds transgenic system for CRISPR/dCas9 modulation of enhancers and non-coding RNAs in zebrafish}

1

Vanessa Chong-Morrison $^{1,3}$, Filipa C. Simões ${ }^{1,2}$, Upeka Senanayake ${ }^{1}$, Dervla S. 4 Carroll $^{1}$, Paul R. Riley ${ }^{2}$ and Tatjana Sauka-Spengler ${ }^{1, *}$

${ }^{1}$ University of Oxford, Weatherall Institute of Molecular Medicine, Radcliffe Department of 6 Medicine, Oxford OX3 9DS, United Kingdom

${ }^{2}$ University of Oxford, Department of Physiology, Anatomy and Genetics, Oxford OX1 3PT, 8 United Kingdom

${ }^{3}$ Current address: University College London, Department of Cell \& Developmental Biology, $\quad{ }_{10}$ London WC1E 6BT, United Kingdom

*Corresponding author: tatjana.sauka-spengler@imm.ox.ac.uk

Keywords: zebrafish, maize, Ac/Ds, CRISPR, enhancer, non-coding 


\section{Summary statement}

We adapted the Ac/Ds transposition system, which enables continuous expression of guide

\section{Abstract}

Due to its genetic amenability coupled with recent advances in genome editing, the zebrafish 20 serves as an excellent model to examine the function of both coding and non-coding elements. 21 Recently, the non-coding genome has gained prominence due to its critical role in development 22 and disease. Here, we have re-purposed the Ac/Ds maize transposition system to reliably screen 23 and efficiently characterise zebrafish enhancers, with or without germline propagation. We 24 further utilised the system to stably express guide RNAs in microinjected embryos enabling 25 tissue-specific CRISPR/dCas9-interference (CRISPR $i$ ) knockdown of lncRNA and enhancer 26 activity without disrupting the underlying genetic sequence. Our study highlights the utility of 27 Ac/Ds transposition for transient epigenome modulation of non-coding elements in zebrafish. 28 


\section{Introduction}

The non-coding genome has risen to prominence in recent years following successive studies highlighting its numerous roles in development and disease (Krijger and de Laat, 2016, Engreitz 31 et al., 2016). The genome is populated by cis-regulatory elements called enhancers, which are 32 active in a tissue-specific fashion (Rickels and Shilatifard, 2018) and probing their functional 33 relevance requires inactivation in specific cell types and at distinct times. This is particularly 34 important for key developmental regulators often deployed in well-defined spatiotemporal 35 sequences, and thus likely to employ specific enhancers for such activity. Long non-coding 36 RNAs, or lncRNAs, are defined as transcripts >200bp with no known protein product (Quinn 37 and Chang, 2015). Unlike in the case of their protein-coding counterparts, functional studies to 38 dissect lncRNA function can be challenging due to limiting factors such as their under-characterisation, low expression levels, short transcript length and rapid degradation. $\quad 40$ Furthermore, antisense lncRNAs that overlap protein-coding loci but are transcribed on the ${ }_{41}$ opposite strand are often found within important developmental loci (Bassett et al., 2014). 42 Uncoupling their function from that of their cognate genes represents a major obstacle in the ${ }_{43}$ experimental design of relevant knockout studies.

In vitro methods for interrogation of non-coding RNAs and enhancers are useful but may $\quad 45$ not recapitulate results obtained from in vivo transgenic/knockout models, which, on the other 46 hand, are often time-consuming. In this study, we have sought to bridge this gap by developing 47 an efficient and flexible molecular toolkit to functionally assay non-coding elements in the 48 zebrafish using transient and quantifiable in vivo approaches. The toolkit was based on 49 re-purposing the maize transposon system first identified by Barbara McClintock in the late 50 1940s (McClintock, 1950). Molecular characterisation of the system led to identification of the 51 components required for transposition to occur - two Ds (Dissociation) genetic elements and an 52 Ac (Activator) transposase (Fedoroff et al., 1983). Buoyed by this important finding, Ac/Ds 53 elements were employed to facilitate the integration of a reporter construct into the zebrafish 54 genome with high efficiency, leading to remarkable germline transmission rates (Emelyanov 55 et al., 2006). This approach was the integration method of choice used to generate transgenic 56 
zebrafish lines for the chemical-inducible LexPR transactivation system (Emelyanov and Parinov, 2008, Kenyon et al., 2018), as well as for a systematic mutagenesis gene-trapping potential for transient expression of DNA elements in $\mathrm{F}_{0}$ embryos, which is a current limitation ${ }_{61}$ of the zebrafish model. Several other genome integration methods currently exist in vertebrates 62 (Kawakami, 2007, Vrljicak et al., 2016). In particular in the zebrafish, Tol2-mediated genomic ${ }_{63}$ transposition is an established approach for somatic and germline integration of DNA constructs and is almost always the method-of-choice for generating transgenic reporter lines. For transient DNA integration experiments, however, Tol2-mediated transposition often produces variable results with a high rate of $\mathrm{F}_{0}$ embryos displaying non-specific background and/or mosaic expression. The analysis of exogenous gene expression and testing of enhancer/reporter activity therefore often relies on the generation of $F_{1}$ offspring carrying relevant constructs. In this study, we expanded the use of Ac/Ds-mediated integration in zebrafish to test and validate putative cis-regulatory elements in transient, as an alternative to the Tol2-integration-based approach. This redirects the focus of previous zebrafish Ac/Ds-integration studies from germline propagation efficiency to characterisation of its utility for somatic integration-based experiments.

To efficiently probe non-coding element function in $\mathrm{F}_{0}$ embryos in zebrafish, we have used tissue-specific epigenome engineering. Other knockdown approaches in injected $\mathrm{F}_{0}$ zebrafish embryos currently exist, such as morpholino-mediated obstruction of protein synthesis or RNA-splicing, or somatic gene editing using TALENs or CRISPR/Cas9. However, these approaches lack spatiotemporal specificity and, as a result, render it difficult to distinguish between a cell-specific phenotype and secondary effects resulting from ubiquitous knockdown of the gene-of-interest. CRISPR/dCas9-based interference (CRISPR $i$ ) utilises nuclease-deficient Cas9 (dCas9) fused to transcriptional regulator domains which are targeted to specific genomic 82 regions using "guide RNAs" (sgRNAs). To inhibit transcription, dCas9 can be targeted to 83 transcription start sites (TSS) of genes to inhibit RNA Polymerase II by steric hindrance (Qi ${ }_{84}$ et al., 2013), or fused to effector domains such as Kruppel-associated box (KRAB) (Gilbert 85 
et al., 2013) or four concatenated mSin3 repressive domains (SID4x) (Konermann et al., 2013) 86 to induce chromatin changes inhibitive of transcription. Crucially, as CRISPR $i$ complexes are 87 directed to the TSS of target genes, this allows the perturbation of gene expression without 88 modifying the endogenous locus sequence, making it a well-suited tool to study the function of 89 non-coding genes (Thakore et al., 2015, Konermann et al., 2015, Liu et al., 2016, Williams et al., 90 2018). Similarly, ectopic activation of genes can be achieved by CRISPR $a$ in specific cell types 91 using the VP64 activator domain (Mali et al., 2013). The caveat to using these approaches in 92 the zebrafish embryo is the need for extended expression of sgRNAs, which is limited by the 93 current gold-standard of injecting in vitro-transcribed sgRNAs. In the absence of Cas9 protein, 94 uncapped and non-polyadenylated sgRNAs degrade quickly (Mir et al., 2018). Therefore such 95 experiments can only be performed at the 1-cell stage and either have to be analysed during the $\quad 96$ first 24-48 hours of development or sgRNA expression needs to be propagated to the germline. 97 Here, in addition to building zebrafish BAC transgenic lines expressing CRISPR $i / a$ effectors 98 dCas9-SID4x and dCas9-VP64 in a tissue-specific fashion, we also generated and employed 99 Ac/Ds-integrated DNA constructs to deliver small guide RNAs constitutively in order to 100 transiently modulate the activity of non-coding elements in transgenic $\mathrm{F}_{0}$ embryos in vivo. 101

Our Ac/Ds toolkit broadens the potential of the zebrafish embryo for rapid studies of 102 non-coding genomic elements. By harnessing its reliability and efficiency for somatic 103 integration, the toolkit can be robustly used either in transient and/or to complement 104 transgenic-based methods.

\section{Results and Discussion}

\section{Ac/Ds transposition is more effective than Tol2 for transient integration of transgenes in $\mathbf{F}_{0}$ zebrafish embryos.}

As a first step in re-purposing the maize Ac/Ds transposition system for zebrafish, we generated 109 a new enhancer/reporter construct (pVC-Ds-E1b:eGFP-Ds) for efficient and reproducible in 110 vivo testing of enhancer activity. The construct consists of the eGFP expression cassette under ${ }_{111}$ the control of the zebrafish E1b minimal promoter (Becker et al., 2016) flanked by Ds elements 112 
for integration into the genome (Emelyanov et al., 2006, Emelyanov and Parinov, 2008), and 113 features a multiple cloning site for cloning enhancer sequences to test upstream of the minimal 114 promoter (Fig.1A). We compared the efficiency and efficacy of Ac/Ds- and Tol2-mediated 115 integration (Kawakami, 2004) by transiently expressing a previously predicted zebrafish 116 enhancer for pax3a with strong activity (Trinh et al., 2017, Gavriouchkina et al., 2017) ("pax3a 117 enhancer"). Ac/Ds and Tol2 versions of the pax3a enhancer were microinjected into 118 Gt(FoxD3:mCherry) ${ }^{\text {ct110aR }}$ transgenic embryos (Hochgreb-Hägele and Bronner, 2013) together 119 with $24 \mathrm{pg}$ Ac or 50-80 pg Tol2 mRNA, respectively. We observed a clean and tissue-specific 120 pattern of eGFP expression in neural crest cells at the neural plate border with $30 \mathrm{pg}$ of the ${ }_{121}$ Ac/Ds vector, a result that could only be recapitulated with $150 \mathrm{pg}$ of Tol2 vector (Fig.1A), ${ }_{122}$ indicating higher somatic integration efficiency by Ac/Ds as previously reported (Vrljicak et al., ${ }^{123}$ 2016). To quantify this observation, we collected embryos injected with $30 \mathrm{pg}$ of the Ac/Ds or ${ }_{124}$ Tol2 pax3a enhancer for GFP immunohistochemistry (IHC) together with Hoechst-staining of 125 nuclei, followed by confocal imaging and Imaris image analysis (Supp.Fig.1, Materials \& 126 Methods). Ac/Ds-injected embryos demonstrated up to 9-fold increase in $\mathrm{GFP}^{+} / \mathrm{Hoechst}^{+}{ }_{127}$ signal compared to Tol2-injected embryos $(\mathrm{p}=0.001)$ (Fig.1B). The lower load requirement for ${ }_{128}$ Ac/Ds (30 pg per embryo) to yield efficient integration also resulted in much lower mosaicity 129 within a clutch of injected embryos (64 to $95 \%$ of injected embryos with distinguishable 130 expression pattern, compared to $<40 \%$ for Tol2) and therefore provided an efficient binary ${ }_{131}$ yes/no tool for transient screening of enhancer activity. To assess the limitations of 132 Ac/Ds-integration in $\mathrm{F}_{0}$, we next compared Ac/Ds and Tol2 versions of a previously predicted ${ }_{133}$ enhancer for sox10 with weak activity (scattered labelling of few neural crest cells with either ${ }_{134}$ method) (Trinh et al., 2017, Gavriouchkina et al., 2017) ("sox10 enhancer") using the same 135 quantification approach (Supp.Fig.2). Tol2-injected embryos demonstrated more mosaic ${ }_{136}$ intra-clutch GFP expression pattern compared to Ac/Ds-injected embryos, with a higher ${ }_{137}$ proportion of embryos without any specific detectable $\mathrm{GFP}^{+}$cells that were excluded from 138 quantification. Conversely, although weak "sox10 enhancer" showed smaller number of GFP ${ }^{+}{ }_{139}$ cells per embryo, Ac/Ds-integration approach allowed us to detect reproducible and consistent 140 neural crest-specific activity of this enhancer. While Ac/Ds- and Tol2-mediated integration of ${ }_{141}$ 
the 'weak' enhancer resulted in comparable $\mathrm{GFP}^{+} / \mathrm{Hoechst}^{+}$signal, a much higher variability ${ }_{142}$ between embryos was observed by the Tol2-mediated approach (Fig.1C).

These results demonstrated a clear advantage of Ac/Ds- over Tol2-mediated enhancer testing, 144 and in general transient expression systems using DNA constructs in zebrafish, as Ac/Ds ${ }_{145}$ transposition yielded a higher number of construct integrations resulting in lower cell mosaicity 146 rate. This was particularly notable with a strong element such as the pax3a enhancer, where a ${ }_{147}$ high number of cells showing consistent enhancer activity pattern were successfully labelled. ${ }_{148}$ Furthermore, the utility of Ac/Ds in being able to inject both DNA and mRNA at lower amounts is significant. Toxicity issues associated with the higher levels required for Tol2-based 150 somatic integration could be avoided, and lower amounts of DNA required for high integration 151 efficiency minimises ectopic activity from episomal expression of non-integrated plasmid. This 152 places the zebrafish embryo on par with the chick embryo as an excellent model for testing 153 enhancer activity transiently in injected $\mathrm{F}_{0}$ embryos (Streit et al., 2013). Taken together, we ${ }_{154}$ demonstrated that Ac/Ds-mediated integration is not only more efficient for transgenes with 155 strong activity but at the same time more efficacious for transgenes with weaker activity. 
bioRxiv preprint doi: https://doi.org/10.1101/450684; this version posted October 23, 2018. The copyright holder for this preprint (which was not certified by peer review) is the author/funder, who has granted bioRxiv a license to display the preprint in perpetuity. It is made available under aCC-BY-NC-ND 4.0 International license.

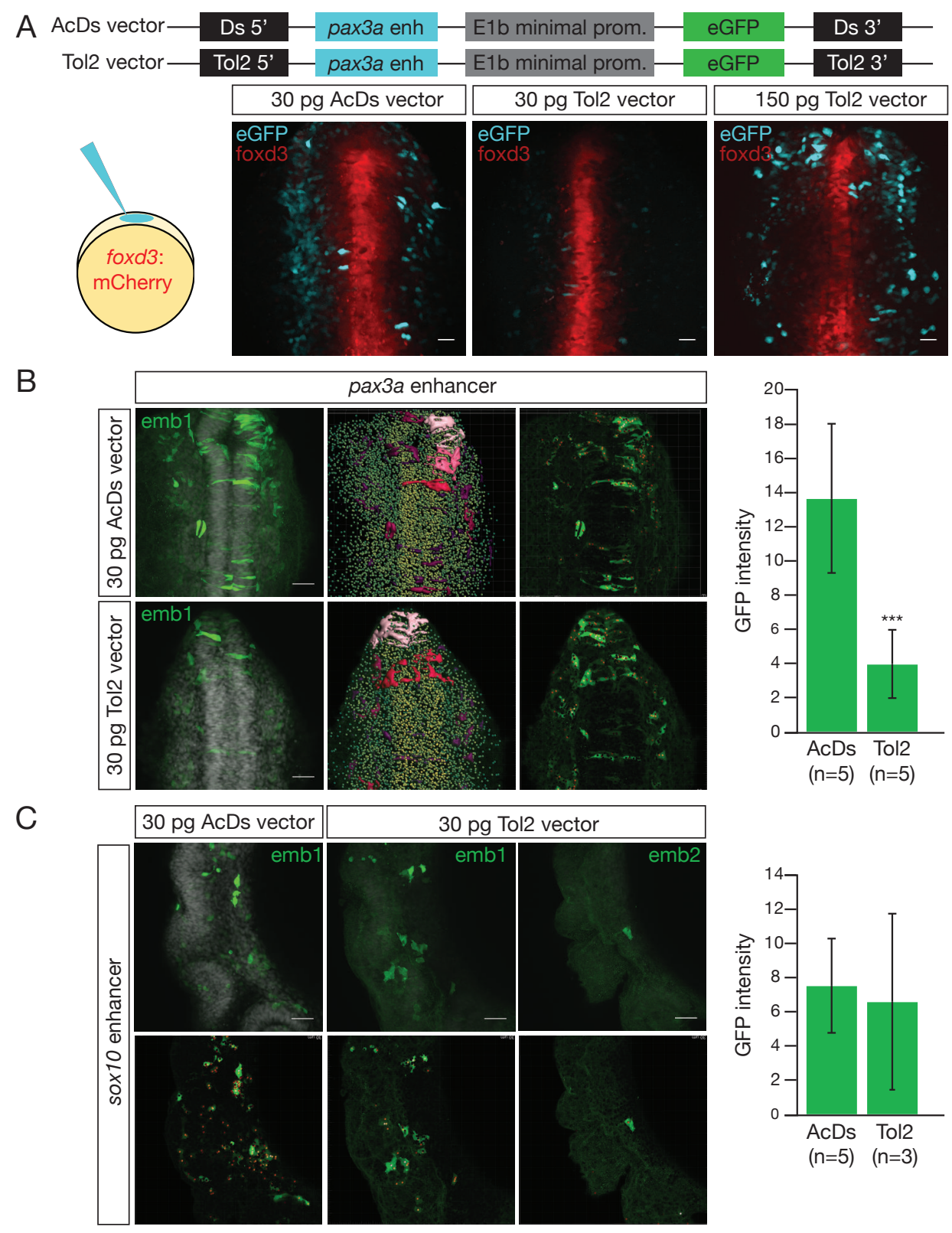

Figure 1. Ac/Ds integration is more effective than Tol2 in $\mathrm{F}_{0}$ embryos.

A Schematic of constructs containing a neural crest pax3a enhancer (cyan) positioned upstream of the zebrafish E1b minimal promoter (grey) and driving eGFP (green) expression harbouring either Ds- or Tol2-integration arms (black). $30 \mathrm{pg}$ of either construct ("Ac/Ds" and "Tol2") was microinjected into Gt(FoxD3:mCherry)ct110aR embryos (to visualise neural tube) together with Ac or Tol2 mRNA, respectively. Live confocal imaging highlighted similar levels of neural crest cell labelling between $30 \mathrm{pg}$ Ac/Ds and $150 \mathrm{pg}$ Tol2, but $30 \mathrm{pg}$ Tol2 injections yielded much weaker signal in comparison. B Labelling efficiencies of the Ac/Ds and Tol2 pax3a enhancer were quantified using immunohistochemistry and Imaris. Ac/Ds resulted in up to 9-fold increase (Student $t$-test; $p=0.001$ ) of $\mathrm{GFP}^{+} /$Hoechst $^{+}$signal compared to Tol2. C The same approach in (B) was used to compare labelling efficiency of a sox10 enhancer with weak activity. Although both versions resulted in comparable $\mathrm{GFP}^{+} / \mathrm{Hoechst}^{+}$signal, Ac/Ds demonstrated less variability compared to Tol2. Scale bar: $40 \mu \mathrm{m}$. 
In vivo characterisation of novel neural crest enhancers using Ac/Ds. embryos were comparable to Tol2, consistent with previous reports (Emelyanov et al., 2006, 162 Emelyanov and Parinov, 2008). We also characterised three additional enhancers - one 163 predicted for pax3a and two for sox10 (Fig.2; pax3a_E4, sox10_E5 and E7) in both $\mathrm{F}_{0}$ and ${ }_{164}$ following germline transmission (pax3a_E4), or in $\mathrm{F}_{0}$ only (sox10_E5 and E7) (Supp.Fig.3). ${ }_{165}$ Pax3a and sox10 are well-characterised transcription factors with known roles in neural crest ${ }_{166}$ development (Alkobtawi et al., 2018). Pax3a_E4 and E5 are located 23.5 and 6kb upstream of 167 the transcription start site (TSS) of pax3a at open chromatin regions detected by ATAC-seq ${ }_{168}$ (Buenrostro et al., 2013) performed on FAC-sorted neural crest cells (Trinh et al., 2017, 169 Gavriouchkina et al., 2017) (Fig.2A, maroon track). Sox10_E2, E5 and E7 are located 33, 13kb 170 upstream of the TSS and 3.7kb downstream of the 3'UTR of sox10, respectively (Fig.2B, ${ }_{171}$ maroon track).

To test the co-localisation of pax3a expression with pax3a_E4 or pax3a_E5 activity, we utilised the Hybridisation Chain Reaction (HCR) method (Choi et al., 2018) to detect eGFP and endogenous pax3a mRNAs. At 6 somite stage (ss), pax3a is strongly expressed in 175 premigratory neural crest at the neural plate border region (Fig.2A, cartoon reproduced from 176 Zfin in situ data). Co-localisation of $e G F P$ and pax3a mRNA transcripts in this region were 177 detected in $\mathrm{F}_{0}$-injected embryos, and this result was recapitulated in $\mathrm{F}_{2}$ embryos, 178 Tg(pax3a_enh5-E1b:eGFP) ${ }^{\text {ox163 }}$ (Fig.2A). Pax3a_E4 gave a similar result but with overall 179 weaker eGFP expression in both $\mathrm{F}_{0}$ and $\mathrm{F}_{2}, T g\left(\right.$ pax3a_enh4-E1b:eGFP) ${ }^{\text {ox162 }}$ (Supp.Fig.3). ${ }_{180}$

Next, we tested the co-localisation of Sox10 expression with sox10_E2, E5 and E7 activity 181 using immunohistochemistry to detect eGFP and endogenous Sox10 proteins. At 18 ss, sox10 is 182 strongly expressed in migratory neural crest within the cranial region (Fig.2B, cartoon 183 reproduced from (Gavriouchkina et al., 2017)). All sox10 enhancers demonstrated weak but ${ }_{184}$ Sox10-specific activity in $\mathrm{F}_{0}$-injected embryos (Fig.2B, Supp.Fig.3). Remarkably, 185 
sox10_E2-driven eGFP expression dramatically improved following germline transmission with a 186 much higher number of $\mathrm{GFP}^{+} / \operatorname{Sox}_{10}{ }^{+}$cells being detected in Tg(sox10_enh2-E1b:eGFP) ox120 ${ }_{187}$ $\mathrm{F}_{2}$ embryos (Fig.2B). Interestingly, this "germline-boosting" effect was not observed in five 188 other enhancer lines that we have propagated to the germline.

To recover integration sites of the transgene in a genome-wide fashion, we performed 190 splinkerette-PCR-NGS on a pool of $\mathrm{GFP}^{+} \mathrm{F}_{2}$ embryos (Supp.Fig.4, Materials \& Methods). ${ }_{191}$ High-confidence sites were defined as genomic regions where the signal of mapped reads from 192 splinkerette PCR amplicons representing genomic regions flanking Ds-5' and 3' arms were 193 enriched over background (qval<0.01) (Fig.2B', splink Rv and splink Fwd tracks). We 194 identified 3,521 high-confidence sites, significantly higher than a previous study reporting $1,685 \quad 195$ integration sites across 424 Ac/Ds transgenic lines (Vrljicak et al., 2016). We reasoned that our 196 result may represent most of the initial integration events still present in the $\mathrm{F}_{1}$ germline, as ${ }_{197}$ indicated by the 5,473 putative inserts identified in $\mathrm{F}_{0}$-injected embryos by Vrljicak et al.. 198 Echoing the previous study, we also found a large proportion of integration sites (88.2\%) to 199 overlap annotated repeat elements (Fig.2B', pie chart) and enriched at gene regions with broad 200 distribution across promoters, introns, exons and transcription termination sites (TTS) 201 (Fig.2B', red and purple bar chart) (Vrljicak et al., 2016). 202

The results demonstrated by the weak sox10_E2 enhancer fortuitously highlighted the 203 potential for our approach to uncover candidates that would otherwise have been overlooked at 204 the $\mathrm{F}_{0}$ screening stage if Tol2-integration was used. The higher likelihood of a false positive 205 result with Tol2 could influence a negative decision for the propagation of the transgene as a 206 reporter line, thus eliminating potential lines that could be biologically relevant. In short, we 207 showed that the Ac/Ds enhancer construct is a robust and useful tool to characterise putative ${ }_{208}$ enhancers with both strong and weak activity. 
bioRxiv preprint doi: https://doi.org/10.1101/450684; this version posted October 23, 2018. The copyright holder for this preprint (which was not certified by peer review) is the author/funder, who has granted bioRxiv a license to display the preprint in perpetuity. It is made available under aCC-BY-NC-ND 4.0 International license.
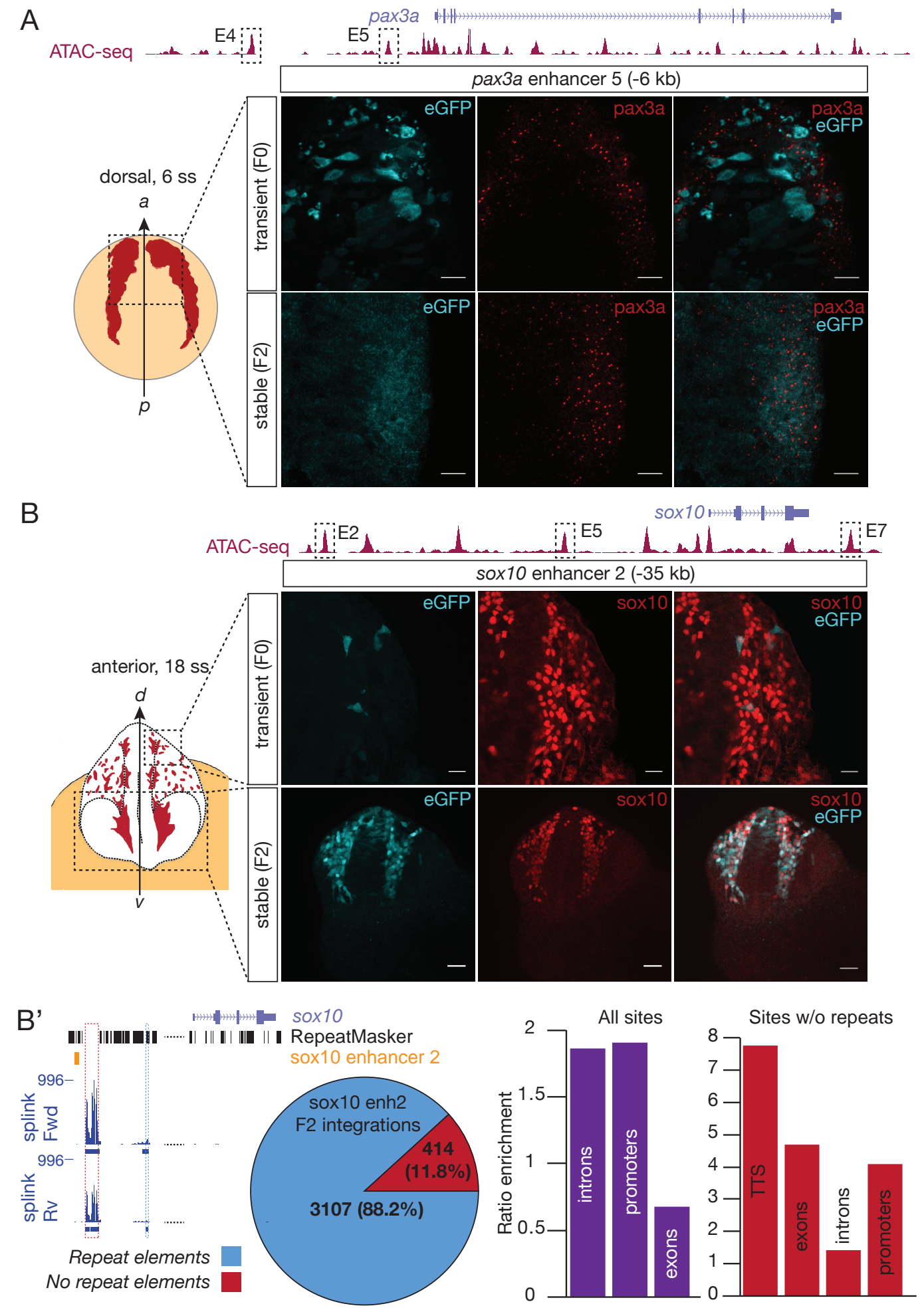

Figure 2. In vivo characterisation of pax3a and sox10 enhancers using Ac/Ds integration. Full legend on next page. 
A Two putative pax3a enhancers (E4 and E5; E5 also shown in Fig1) are visualised on UCSC ${ }_{211}$ Genome Browser (maroon, ATAC-seq track; indigo, pax3a gene locus). E5-driven eGFP 212 transcripts in $\mathrm{F}_{0}$ and $\mathrm{F}_{2}$ embryos demonstrated a similar expression pattern that overlapped 213 endogenous pax3a mRNA. Note the post-fixing eGFP protein signal detected in $\mathrm{F}_{0}$ embryo. B ${ }_{214}$ Three putative sox10 enhancers (E2, E5 and E7; E2 also shown in Fig1) are visualised on 215 UCSC Genome Browser (maroon, ATAC-seq track; indigo, sox10 gene locus). EGFP and ${ }_{216}$ endogenous Sox10 proteins were detected using immunohistochemistry. Weak E2-driven eGFP ${ }_{217}$ activity labelled very few Sox10-positive cells in $\mathrm{F}_{0}$ embryos. $\mathrm{F}_{2}$ embryos demonstrated a 218 remarkably higher number of Sox10-positive cells with eGFP activity. B' Integration sites of ${ }_{219}$ the sox10_E2:eGFP transgene in $\mathrm{F}_{2}$ embryos identified using splinkerette-PCR-NGS. Reads 220 corresponding to flanking genomic regions on Ds-3' end (navy blue; splink Fwd) and Ds-5' end 221 (navy blue; splink Rv) were mapped to the zebrafish genome (GRCz10) and peaks corresponding ${ }_{222}$ to integration sites were bioinformatically called (navy blue boxes). The sox10 locus is shown 223 as an example, with the position of sox10_E2 highlighted in orange. 88.2\% of integration sites 224 partially/fully overlapped annotated repeat elements (blue; highlighted with dotted box), while 225 the remaining $11.8 \%$ (red; highlighted with dotted box) did not. Annotated introns, promoters 226 and exons were enriched $(p<0.04)$ within all integration sites (purple bar chart). Transcription 227 termination sites (TTS) were also enriched $(p<0.01)$ within integration sites that did not ${ }_{228}$ overlap annotated repeat elements (red bar chart). Scale bar: $20 \mu \mathrm{m} ; 40 \mu \mathrm{m}$ (B-stable (F2)). ${ }^{229}$

\section{Ac/Ds successfully expresses sgRNAs for transient tissue-specific CRISPR $i$.}

The ability to knockdown non-coding elements in vivo is essential for the dissection of their ${ }_{231}$ function. Currently, the most commonly used method to deliver sgRNAs for CRISPR/Cas9 in 232 zebrafish is via microinjection of in vitro-transcribed sgRNAs into single cell embryos. While ${ }_{233}$ cost-effective and straightforward, this approach risks decreasing the efficiency for 234 CRISPR-mediated events in the embryo, as the uncapped and non-polyadenylated nature of sgRNAs renders them sensitive to degradation in vivo (Mir et al., 2018). This is particularly pertinent if the desired goal is to perform CRISPR experiments in a tissue-specific fashion, as unprotected sgRNAs injected in the absence of Cas9 protein or Cas9 mRNA are likely to be development.

We took advantage of Ac/Ds to generate a constitutive sgRNA expression system that 
expression of sgRNAs (Yin et al., 2015) into a custom-made Ac/Ds mini-vector (Fig.3A, ${ }_{245}$ Materials \& Methods). The 20bp spacer region within the cassette was flanked by BsmBI 246 restriction sites to facilitate GoldenGate (Clarke et al., 2012)-like cloning of different sgRNAs $\quad 247$ (Supp.Fig.5A). We compared continuous expression of a scrambled sgRNA sequence from the 248 integrated Ac/Ds-U6a:sgRNA vector (pVC-Ds-DrU6a:sgRNA-Ds) and an in vitro-transcribed 249 sgRNA transcript by injecting $50 \mathrm{pg}$ of vector (with $24 \mathrm{pg} A c$ mRNA) or $80 \mathrm{pg}$ of transcript into 250 wild type embryos, followed by RT-PCR at 5 hours post injection (hpi), 24 hpi and 5 days post 251 injection (dpi). We found that sgRNA expression up to 5 dpi could only be detected using 252 Ac/Ds (Fig.3A). To achieve tissue-specific (neural crest) expression of dCas9 fused to repressive 253 effector protein (SID4x) for CRISPR/dCas9-interference (CRISPR $i$ ) at target regions (Fig.3B), 254 we generated BAC transgenic line TgBAC(sox10:dCas9-SID4x-2a-Citrine)ox117 ("ox117") by 255 taking advantage of the previously validated sox10 BAC clone (Trinh et al., 2017). This 256 approach enabled neural crest-specific expression of dCas9-SID4x in an endogenous sox10-like 257 fashion, under the control of regulatory elements embedded within the sox10 regulatory locus 258 contained in the BAC clone (Fig.3C). 259

We reasoned that the small size of the Ac/Ds-U6a:sgRNA mini-vector $(<4.5 \mathrm{~kb})$, coupled 260 with the small load required for activity, would permit pooling of multiple sgRNAs. We tested a 261 load of up to $160 \mathrm{pg}$ DNA per embryo, where the survival rate was $\sim 50 \%$ in our hands. ${ }_{262}$ Multiplexing sgRNAs is crucial for CRISPR $i$, given previous studies demonstrating the 263 requirement for multiple sgRNAs to illicit successful knockdown (Qi et al., 2013, Williams et al., 264 2018). Previous evidence has also highlighted CRISPRi's potential for strand-specific 265 mode-of-action when dCas9 is used to target transcription elongation, but not initiation, using 266 sgRNAs that bind the non-template strand (Qi et al., 2013). To explore this possibility in the ${ }^{267}$ zebrafish, we microinjected incrossed ox117 embryos with an Ac/Ds-sgRNA pool containing five ${ }_{268}$ sgRNAs that target the TSS of STRG.15268.1, an antisense transcript overlapping sox $9 a \quad 269$ ("sox $9 a$ lncRNA"), while avoiding targeting of sox $9 a$ elongation (Supp.Fig.5B). The sox $9 a \quad{ }_{270}$ lncRNA was identified by de novo assembly (Pertea et al., 2015) of previously published neural ${ }^{271}$ crest RNA-seq datasets (Trinh et al., 2017) and is preceded by a strong ATAC-seq peak at its 5' 272 terminus, indicative of promoter activity (Buenrostro et al., 2013) (Fig.3D, maroon track). ${ }_{273}$ 
A major obstacle to the study of lncRNAs is their significantly lower levels of expression 274 compared to their protein-coding counterparts (Derrien et al., 2012). To circumvent this issue 275 and quantify sox $9 a$ lncRNA levels following CRISPR $i$, we performed semi-quantitative 276 transcript-specific RT-PCR with $\beta$ actin as endogenous control (Fig.3D). To quantify sox $9 a \quad 277$ while taking into account potential strand-unspecific effects, sox $9 a$ was reverse-transcribed 278 using a gene-specific primer targeted at its 3'UTR and localised upstream of the five sgRNAs 279 targeting the TSS of sox $9 a$ lncRNA. Transcript levels were measured using qPCR as per 280 standard practice (Fig.3D). We found modest knockdown $(p=0.03)$ of sox $9 a$ lncRNA in the 281 presence of sgRNAs compared to scrambled sgRNAs or uninjected controls (Fig.3D'), while ${ }_{282}$ sox $9 a$ transcript levels were unaffected in the same samples (Fig.3D"). These findings were 283 consistent with our observation that injected embryos were morphologically normal and support 284 recent evidence that lncRNAs were largely dispensable for development (Goudarzi et al., 2018). 285

In short, we demonstrated that re-purposing of the Ac/Ds approach enabled constitutive 286 expression of sgRNAs in embryos in vivo following genomic integration of the construct into ${ }^{287}$ somatic cells. By pooling multiple sgRNAs, we also provided proof-of-principle evidence that ${ }_{288}$ CRISPR $i$ could be achieved in a strand-specific fashion on a locus with overlapping antisense ${ }_{289}$ transcription. As an aside, we have also generated modified versions of the Ac/Ds-sgRNA 290 vector containing RNA scaffolds for KRAB transcriptional repression (Zalatan et al., 2015) and 291 for the synergistic transcription activation mediator (SAM) system (Konermann et al., 2015). 292 The latter can be coupled with the TgBAC(sox10:Cas9m4-VP64-2a-Citrine) ${ }^{\text {ox118 }}$ line we have 293 also generated, to enable for CRISPR/dCas9-activation (CRISPR $a$ ) of targeted loci (Mali et al., 294 2013) (Supp.Material). 
bioRxiv preprint doi: https://doi.org/10.1101/450684; this version posted October 23, 2018. The copyright holder for this preprint (which was not certified by peer review) is the author/funder, who has granted bioRxiv a license to display the preprint in perpetuity. It is made available under aCC-BY-NC-ND 4.0 International license.
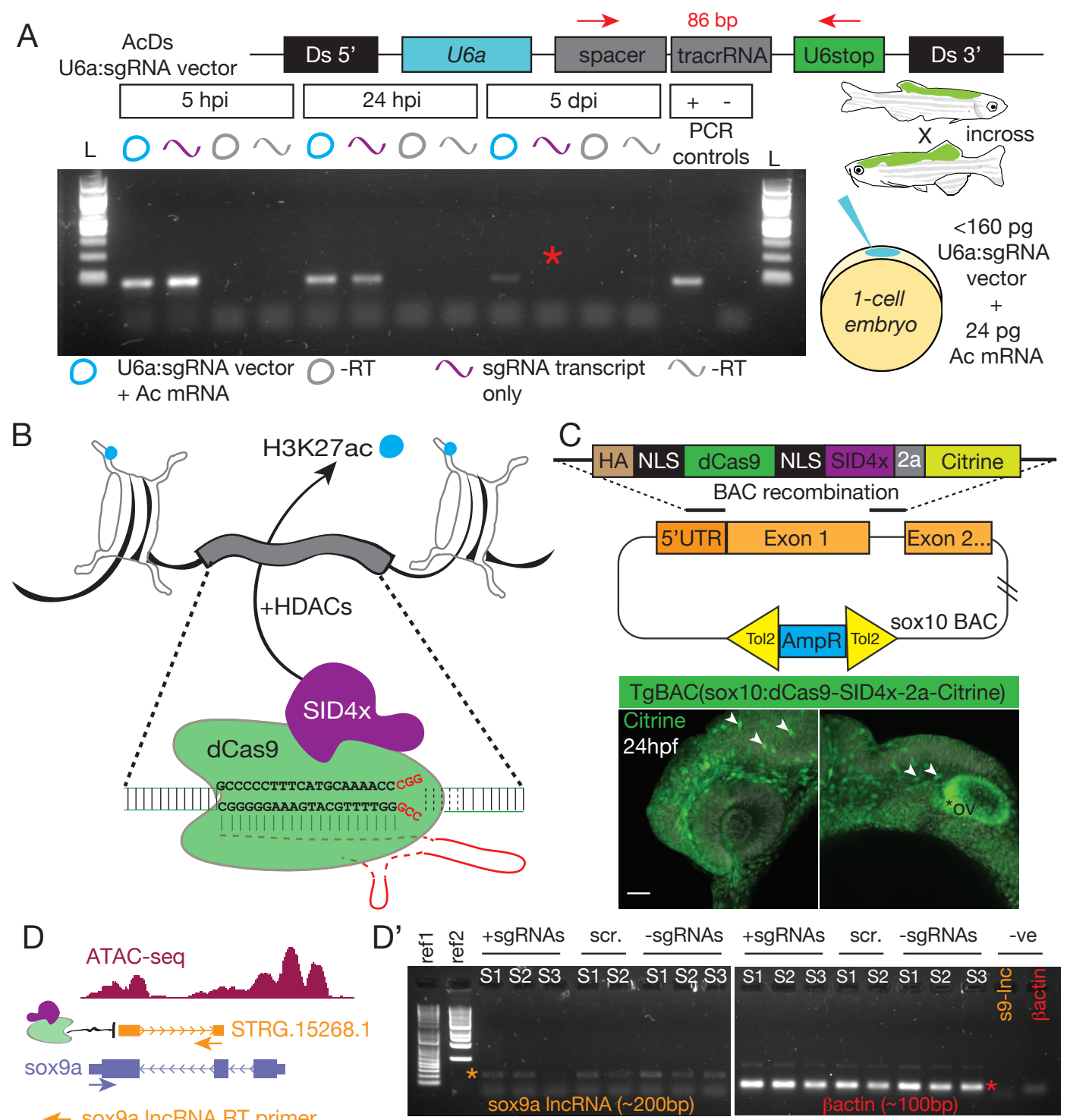

$\leftarrow$ sox9a IncRNA RT primer

(+ $\beta$ actin RT primer)

Reverse transcription $\downarrow$

Limited cycle PCR $\downarrow$

Gel quantification

$\rightarrow$ sox9a transcript RT primer (+ $\beta$ actin RT primer) $\downarrow$ Reverse transcription $\downarrow$ qPCR
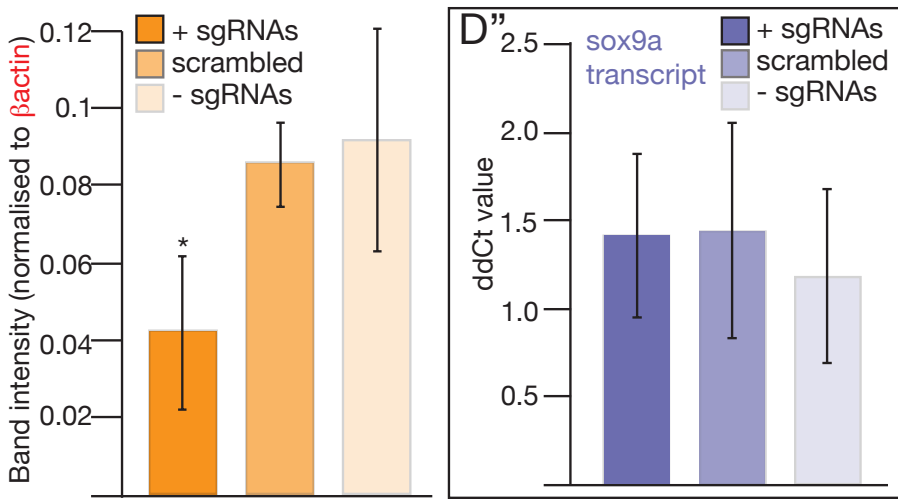

Figure 3. Ac/Ds ubiquitous expression of sgRNAs for Sox10-specific CRISPR $i$ in the neural crest of transgenic $\mathrm{F}_{0}$ embryos. Full legend on next page. 
A A zebrafish U6a promoter is used to drive expression of an sgRNA cassette consisting of spacer region (20bp target sequence), tracrRNA and a U6 termination sequence (U6stop). The entire transgene is flanked by Ds integration arms (black). AcDs-U6a:sgRNA was microinjected into incrossed ox117 embryos and its sgRNA transcript detected by RT-PCR (red arrows indicate primers; product size 86bp) at 5 hours post-injection (hpi), 24 hpi and 5 days post-injection ( $5 \mathrm{dpi}$ ). In parallel, embryos microinjected with in vitro-transcribed sgRNA (with same sequence as AcDs version) were also accessed. At 5 dpi, sgRNA expression was only detected in embryos microinjected with AcDs-U6a:sgRNA. B Nuclease-deficient Cas9 (green; dCas9) fused to the SID4x repressor domain (violet) is targeted to a region-of-interest using sgRNAs (red). The SID4x domain enables transcriptional repression via chromatin compaction, possibly by recruiting histone deacetylases (HDACs). C A Sox10-specific CRISPR $i$ transgenic line, TgBAC(sox10:dCas9-SID 4x-2a-Citrine) ${ }^{o x 117}$, was generated using BAC recombination and Tol2-mediated transgenesis. A ribosome-skipping TaV-2a peptide (2a) mediates separation of dCas9-SID4x from its Citrine reporter protein. The transgene successfully labels neural crest (white arrows) and the otic vesicle (ov) in vivo. D Five AcDs-U6a:sgRNAs targeting the TSS of STRG.15268.1, a transcript overlapping sox $9 a$ in the opposing strand ("sox $9 a$ lncRNA"), were microinjected into ox117 incrossed embryos. Strand-specific reverse transcription (RT) was performed using primers that bind specifically to the predicted 3' end of sox $9 a$ lncRNA (orange) or the 3'UTR of sox9a mRNA (indigo). The sox $9 a$ mRNA RT primer is positioned upstream of sox $9 a$ lncRNA's TSS target region. In both cases, $\beta$ actin was also primed as an endogenous control. Sox $9 a$ lncRNA and sox $9 a$ mRNA were measured using semi-quantitative PCR and qPCR, respectively. D' Semi-quantitative PCR revealed a modest knockdown (Student $t$-test; $p=0.03$ ) of sox $9 a$ lncRNA in the presence of sgRNAs compared to controls. D" Sox $9 a$ mRNA transcript levels were unaffected by sox9a $\operatorname{lncRNA}$ CRISPR $i$, indicating a strand-specific mode-of-action. Scale bar: $40 \mu \mathrm{m}$.

\section{CRISPRi of sox10 enhancers affects Sox10 expression.}

Finally, we investigated the functional relationship between sox10_E2, E5 and E7 enhancers, ${ }_{323}$ characterised by our Ac/Ds enhancer assay, and endogenous sox10 expression using our optimised CRISPR $i$ approach (Fig.4A), as enhancer effect on gene expression is thought to be 325 additive (Hay et al., 2016, Will et al., 2017). To test the contributions of multiple enhancers to 326 sox10 gene activity, TgBAC(sox10:dCas9-SID4x-2a-Citrine) ${ }^{\text {ox117 }}$ incrossed embryos were 327 injected with a pool of $15 \mathrm{Ac} / \mathrm{Ds}$ sgRNAs targeting all three sox10 enhancers. Embryos were 328 fixed and immunohistochemistry performed to detect Citrine (cells expressing dCas9-SID4x) 329 and nuclei containing endogenous Sox10 protein (Materials \& Methods). We identified three 330 different scenarios in terms of co-expression - (1) dCas9-SID4x ${ }^{+}$only* cells, (2) 331 sox $10^{+} / \mathrm{dCas}-\mathrm{SID} 4 \mathrm{x}^{+* *}$ double-positive cells and (3) Sox $10^{+}$only cells (this scenario was 332 observed due to faint levels of Citrine at the stage of analysis) (Fig.4B). We reasoned that such 333 
a combined outcome and scenario (3) with a decrease in Citrine occurred also due to the fact 334 our approach did not only target sox10_E2, E5 and E7 enhancers within the endogenous sox10 335 locus, but also within the regulatory region included in the ox117 BAC allele driving 336 dCas9-SID4x. As a consequence, Citrine and dCas9-SID4x expression was progressively 337 decreased and with it lessened the effect of epigenome modulation. To quantify this 338 phenomenon, we decided to focus on initial stages dCas9-SID4x activity to avoid the decrease in 339 its activity (and thus release of inhibition) due to the described regulatory conundrum. Three 340 different embryos (+/- Ac/Ds sgRNAs) were subjected to the same immunohistochemistry 341 protocol and imaged; dCas9-SID4x ${ }^{+}$cells were counted on individual slices [i.e. scenario 342 $(1)+(2)]$ and scenario (2), see Materials \& Methods, Supp.Material). The knockdown effect was ${ }_{343}$ calculated as a ratio of Sox $10^{+} / \mathrm{dCas}-\mathrm{SID} 4 \mathrm{x}^{+* *}$ cells in dCas9-SID4x ${ }^{+}\left(*+{ }^{* *}\right)$ counted cells 344 (Fig.4B'). Similar to the sox 9 a lncRNA knockdown experiment, we showed that deactivating 345 these three enhancers modestly diminished Sox10 expression $(p=0.02)$ (Fig.4B'). 346

In conclusion, these results demonstrated the utility of our Ac/Ds approach as an exploratory 347 tool to cell-specifically probe enhancers in order to elucidate their function in vivo, without the 348 need for or prior to laborious generation of stable transgenic lines for further characterisation. 349 


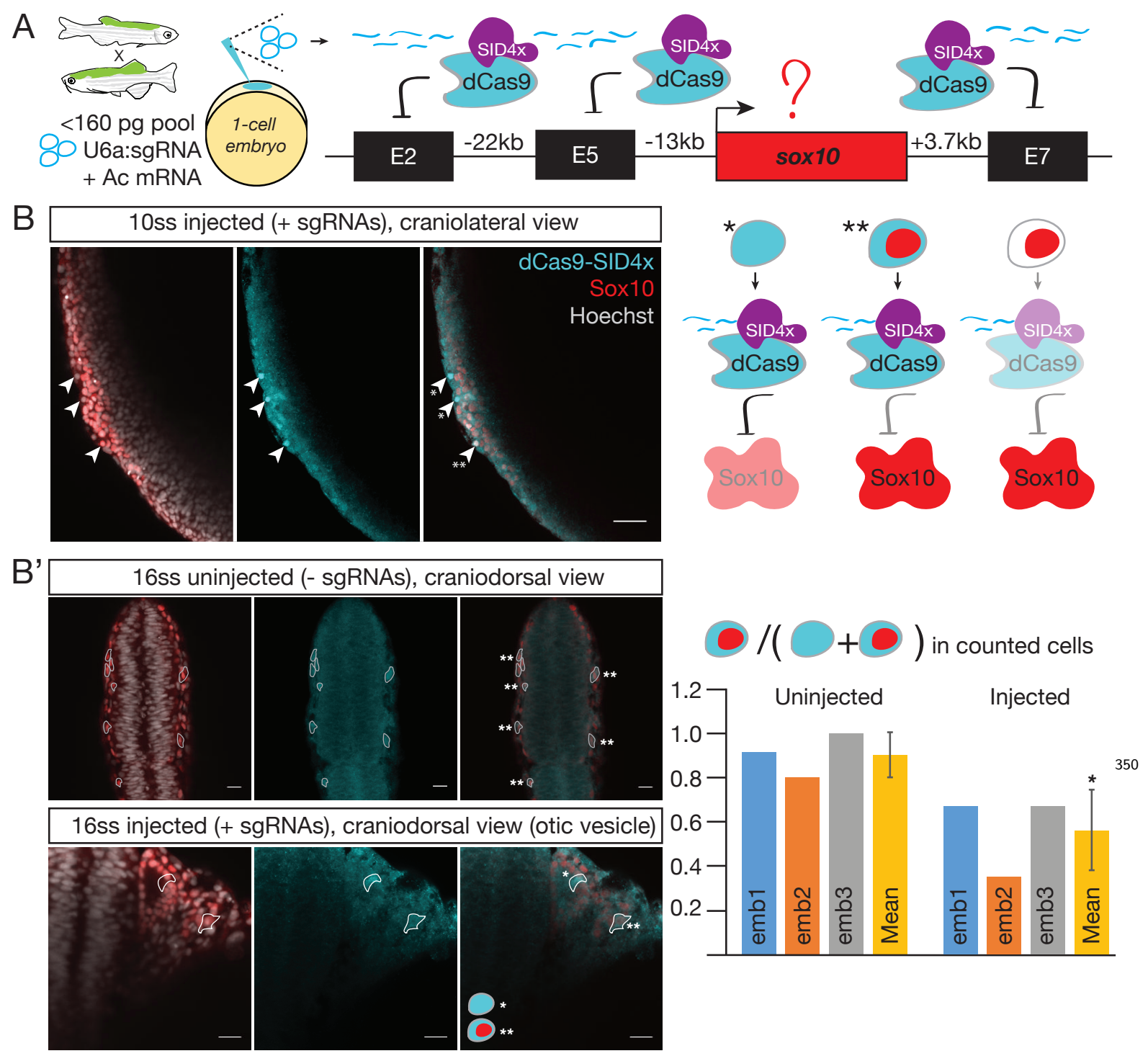

Figure 4. CRISPR $i$ of sox10 enhancers affects Sox10 expression.

A CRISPR $i$ was performed on three sox10 enhancers described earlier (E2, E5 and E7) by simultaneously microinjecting five AcDs-U6a:sgRNAs per enhancer (15 in total) into ox117 incrossed embryos. Immunohistochemistry followed by confocal imaging to detect Citrine and Sox10 proteins was used as a qualitative and quantitative readout of interference. B Two outcomes (*; Sox10 protein undetected and **; Sox10 protein detected) were observed as a result of sox10_E2,E5,E7 CRISPR $i$ in cells where Citrine (ergo dCas9-SID4x) is present, indicating incomplete knockdown and/or secondary effects of the sgRNAs targeting sox10_E2,5,7 that are also present on the dCas9-SID4x BAC allele. B' To quantify this observation, cells with high Citrine expression (dCas9-SID4 $\mathrm{x}^{+}$) were individually counted in two separate slices per embryo across three different embryos per condition. The ratio of Sox $10^{+} / \mathrm{dCas}-\mathrm{SID} 4 \mathrm{x}^{+}$events in dCas9-SID $4 \mathrm{x}^{+}$counted cells revealed a modest decrease (Student $t$-test; $p=0.02$ ) in Sox10 expression following sox10_E2,E5,E7 CRISPRi. Scale bar: $40 \mu \mathrm{m}(\mathbf{B}) ; 20 \mu \mathrm{m}\left(\mathbf{B}^{\prime}\right)$. 


\section{Materials \& Methods}

\section{Zebrafish husbandry}

All zebrafish experiments were conducted according to regulated procedures authorised by the 353 UK Home Office within the framework of the Animals (Scientific Procedures) Act 1986. Wild 354 type and transgenic embryos were derived from $\mathrm{AB}$ or $\mathrm{AB} / \mathrm{TL}$ mix strains.

\section{Plasmids and oligo sequences}

Full lists of plasmids (including Addgene submissions) and oligo sequences are available as 357 Supplementary Material.

\section{Enhancer vector cloning}

Putative enhancer elements were amplified from genomic DNA by PCR and cloned into pVC-Ds-E1b:eGFP-Ds (Addgene ID 102417) linearised with NheI. Cloning was performed using 361 In-Fusion $^{\mathrm{TM}}$ HD Cloning Plus (Takara) or Gibson Assembly cloning (Gibson et al., 2009). 362 Cloning reactions were transformed into chemo-competent cells and plasmids were prepared 363 using QIAprep Spin Miniprep kit (27104, Qiagen) and stored in Elution Buffer. Inserts where 364 verified by Sanger sequencing using T7 primer.

\section{Cloning of Ac/Ds-U6a:sgRNA vector(s)}

We previously generated a mini-vector with Ds arms flanking a multiple cloning site 367 (pVC-Ds-MCS-DS; Addgene ID 102416). The insert consisting of zebrafish U6a promoter, 368 spacer region, tracrRNA, and U6 termination sequence (Yin et al., 2015) was amplified by PCR 369 using a custom-ordered gBlock Gene Fragment (Integrated DNA Technologies) as template. 370 The PCR product was gel-purified and cloned into the mini-vector linearised with SnaBI and 371 NheI, using In-Fusion ${ }^{\mathrm{TM}}$ HD Cloning Plus (Takara). To clone desired sgRNA targets, 372 complementary oligo pairs were ordered as follows: (1) TTCG-5'[20bp target without PAM]3' and 373 (2) AAAC-5' [20bp target without PAM in reverse complement]3' (Supp.Material). $50 \mu \mathrm{M}$ of each 374 oligo were combined in a $50 \mathrm{\mu L}$ reaction and annealed in a thermocycler $\left(94^{\circ} \mathrm{C} 5 \mathrm{mins}\right.$, decrease 375 
to $22^{\circ} \mathrm{C}$ at $1{ }^{\circ} \mathrm{C} / \mathrm{min}, 4^{\circ} \mathrm{C}$ hold). Cloning reaction was prepared by combining $70 \mathrm{ng}$ pVC-Ds-DrU6a:sgRNA-Ds vector, $5 \mathrm{ng}$ annealed sgRNA, 10U of BsmBI and 20U of T4 DNA 377 ligase (M0202, NEB) in 1X T4 DNA Ligase Buffer with final volume $20 \mu \mathrm{L}$. 'GoldenGate' 378 cycling conditions were used as follows: $10 \mathrm{X}\left(37^{\circ} \mathrm{C} 5 \mathrm{mins}, 16^{\circ} \mathrm{C} 10 \mathrm{~min}\right), 50{ }^{\circ} \mathrm{C} 5 \mathrm{mins}, 80^{\circ} \mathrm{C} 5 \quad 379$ mins. $2 \mu \mathrm{L}$ of the reaction was transformed into chemo-competent cells and plated onto 380 Ampicillin plates. Two colonies per sgRNA were screened by Sanger sequencing using U6a 381 promoter primer TCACTCACCACCTCCCAAAA.

\section{Ac transposase mRNA synthesis}

To prepare Ac transposase mRNA, pAC-SP6 (Addgene ID 102418) (Emelyanov et al., 2006) 384 was linearised with BamHI and purified under RNase-free conditions. In vitro transcription was 385 performed using mMESSAGE mMACHINE ${ }^{\mathrm{TM}}$ SP6 Transcription Kit (AM1340, ThermoFisher). 386 mRNA was purified under RNase-free conditions using phenol-chloroform followed by ethanol 387 precipitation and the pellet resuspended in RNase-free water. mRNA quality was assessed by 388 gel electrophoresis (sharp intact band without degradation) and quantified using Qubit ${ }^{\mathrm{TM}} \mathrm{RNA}{ }_{389}$ HS Assay kit (Q32852, ThermoFisher). For long term storage in $-80^{\circ} \mathrm{C}$ the purified mRNA 390 was prepared as $1 \mu \mathrm{L}$ aliquots and limited to one freeze-thaw cycle. Prior to use, an aliquot is 391 freshly diluted with nuclease-free water and the excess discarded after use.

\section{Ac/Ds microinjections}

All microinjections were performed by injecting $2.3 \mathrm{~nL}$ into the blastula of one-cell stage 394 embryos within 5 to 20 mins post fertilisation. To prepare microinjection aliquots, Miniprepped 395 plasmids in Elution Buffer were diluted at least 20-fold with nuclease-free water. The following 396 conditions have been optimised using the Nanoject II system (Drummond Scientific) and may 397 have to be adjusted if using a different microinjector. 
6.2 Ac/Ds-U6a:sgRNA injections for CRISPRi. Single sgRNA injections were sgRNA, up to $160 \mathrm{pg}$ of DNA and $24 \mathrm{pg} A c$ mRNA were injected per embryo (maximum $15 \quad 404$ different $\operatorname{sgRNAs)}$.

\section{Ac vs Tol2 quantification}

Following eGFP (with Hoechst) immunohistochemistry (see Section 14), dorsal views of (Bitplane) as "Spots" and "Surfaces" for Hoechst and GFP channels, respectively. To compute ${ }_{410}$ total GFP intensity $(x)$, GFP Mean Intensity values of "Spots" with $d>0$ ( $d=$ distance from ${ }_{411}$ Hoechst "Spot" to GFP "Surface"), was summed. GFP intensity per $1 \mu^{2}{ }^{2}$ "Surface" Area, y, ${ }_{412}$ was calculated by dividing $x$ with total "Surface" Area detected. GFP intensity for $50{\mu m^{2},}^{4}{ }_{413}$ $y^{*} 50$, was plotted as a bar chart.

\section{Ac/Ds-U6a:sgRNA vector vs in vitro-transcribed sgRNA RT-PCR}

Total RNA was extracted from pools of 11 microinjected embryos per condition (vector, or IVT) ${ }_{416}$ using RNAqueous-Micro Total RNA Isolation Kit (AM1931, ThermoFisher). Reverse 417 transcription (RT) was performed using $0.5 \mu \mathrm{M}$ each of R_sgRNA_scaffold_tail (Supp.Material) ${ }^{418}$ in a $10 \mu \mathrm{L}$ reaction $\left(1 \mu \mathrm{g}\right.$ starting RNA) using SuperScript ${ }^{\text {TM }}$ III Reverse Transcriptase ${ }_{419}$ (18080093, ThermoFisher). Reverse transcription was performed at $55^{\circ} \mathrm{C}$ for 60 mins. Primary ${ }_{420}$ PCR was performed using the following primers: R_sgRNA and F_scrambled1_spacer ${ }_{421}$ (Supp.Material). In a $20 \mu \mathrm{L}$ reaction, $0.1 \mu \mathrm{M}$ per primer was combined with $1 \mu \mathrm{L}$ of template $\quad{ }_{422}$ (reverse transcription reaction) in $1 \mathrm{X}$ standard Taq polymerase PCR reaction. Cycling was ${ }^{423}$ performed as follows: $95^{\circ} \mathrm{C} 5 \mathrm{mins}, 35 \mathrm{X}\left(95^{\circ} \mathrm{C} 30 \mathrm{~s}, 55^{\circ} \mathrm{C} 30 \mathrm{~s}\right), 68^{\circ} \mathrm{C} 30 \mathrm{~s}, 12^{\circ} \mathrm{C}$ hold. Next, $\quad{ }_{424}$ secondary PCR was performed using the following primers: R_nested_sgRNA and ${ }_{425}$ F_nested_scrambled1_spacer (Supp.Material). In a $20 \mu \mathrm{L}$ reaction, $0.1 \mu \mathrm{M}$ per primer was $\quad{ }_{426}$ combined with $1 \mu \mathrm{L}$ of template (primary PCR reaction) in $1 \mathrm{X}$ standard Taq polymerase PCR ${ }_{427}$ reaction. Cycling was performed as follows: $95^{\circ} \mathrm{C} 5 \mathrm{mins}, 23 \mathrm{X}\left(95^{\circ} \mathrm{C} 30 \mathrm{~s}, 55^{\circ} \mathrm{C} 30 \mathrm{~s}\right), 68^{\circ} \mathrm{C} 30 \mathrm{~s}, \quad 428$ 


\section{Generation of CRISPR transgenic lines}

TgBAC(sox10:dCas9-SID4x-2a-Citrine) $)^{o x 117}$ and TgBAC(sox10:Cas9m4-VP64-2a-Citrine)ox118 ${ }_{431}$

(Mali et al., 2013) were generated using BAC recombination followed by Tol2 transgenesis as ${ }_{432}$ previously described (Suster et al., 2011, Trinh et al., 2017). Recombination cassettes were 433 amplified from pGEM-T-Easy-HA-NLS-dCas9-NLS-SID4x-TaV-2a-Citrine-FRT-Kan-FRT and ${ }_{434}$ pGEM-T-Easy-HA-NLS-Cas9m4-NLS-VP64-TaV-2a-Citrine-FRT-Kan-FRT (Supp.Material) to ${ }_{435}$ introduce 50bp homology arms for recombination into the sox10 locus in BAC clone $\quad 436$ DKEY-201F15, by replacing sox10's first exon with the recombination cassette.

\section{Sox10 quantification following sox10_E2-7 CRISPRi}

Following Sox10 and Citrine immunohistochemistry (see section 14), Z-stack images of the anterior craniolateral region were obtained using $2.5 \mu \mathrm{m}$ slices. Two slices (at least $15 \mu \mathrm{m}$ apart) ${ }_{440}$ per embryo were used for analysis. First, cells (or cell clusters) with strongest anti-GFP ${ }_{441}$ (Citrine) signal (ergo dCas9-SID4x) were highlighted. Next, the highlighted GFP signal was ${ }_{442}$ superimposed onto the Hoechst channel to refine dCas9-SID4x ${ }^{+}$cell count. Finally, dCas9-SID4x ${ }^{+} /$Hoechst $^{+}$cells with Sox10 signal were counted. This process was repeated for ${ }_{444}$ all slices used in the analysis.

\section{1. sox9a lncRNA RT-PCR}

Total RNA was extracted from pools of 10 CRISPR $i$-microinjected embryos per condition 447 (+sgRNAs, +scrambled sgRNAs, -sgRNAs) using RNAqueous-Micro Total RNA Isolation Kit ${ }_{448}$ (AM1931, ThermoFisher). Sox9a lncRNA-specific reverse transcription (RT) was performed ${ }_{449}$ using $0.5 \mu \mathrm{M}$ each of R_STRG.15268.1, R2_STRG.15268.1 and bactin_E4_R3 (Supp.Material) in 450 a $10 \mu \mathrm{L}$ reaction (maximising amount of starting RNA) using SuperScript ${ }^{\mathrm{TM}}$ III Reverse ${ }_{451}$ Transcriptase (18080093, ThermoFisher). Reverse transcription was performed at $55^{\circ} \mathrm{C}$ for $60 \quad{ }_{452}$ mins. Sox $9 a$ lncRNA and $\beta$ actin PCR were performed using the following primers: ${ }_{453}$ R2_STRG.15268.1 and R_sox9aE3; beta-actin_E3 and beta-actin_E4 (Supp.Material). In a $20 \mu L{ }_{454}$ 
reaction, $0.2 \mu \mathrm{M}$ per primer was combined with a $1 \mu \mathrm{L}$ of template $\left(1: 10\right.$ dilution of reverse $\quad{ }_{455}$ transcription reaction) in 1X Phusion ${ }^{\mathrm{R}}$ High-Fidelity PCR Master Mix with HF Buffer (M0531, 456 NEB). Cycling was performed as follows: $98^{\circ} \mathrm{C} 5 \mathrm{mins}, 23 \mathrm{X}\left(98^{\circ} \mathrm{C} 30 \mathrm{~s}, 57^{\circ} \mathrm{C} 30 \mathrm{~s}\right), 72^{\circ} \mathrm{C} 30 \mathrm{~s}, \quad{ }^{457}$ $12{ }^{\circ} \mathrm{C}$ hold. Results were analysed on a single $1.5 \%$ agarose gel using the $100 \mathrm{bp}$ band of $1 \mathrm{~kb}$ or ${ }^{458}$ $1 \mathrm{~kb}$ Plus ladder (NEB) as reference. Relative band intensities to selected reference were ${ }_{459}$ quantified using BioRad's Image Lab ${ }^{\mathrm{TM}}$ Software.

Both the total RNA extracted for sox $9 a$ lncRNA RT-PCR as well as the RT protocol were ${ }_{462}$ re-utilised for sox $9 a$-specific reverse transcription. Instead, RT primer combination used were ${ }_{463}$ R_sox9a_3UTR and bactin_E4_R3. QPCR was performed using the $\Delta \Delta \mathrm{Ct}$ method with $300 \mathrm{nM}{ }_{464}$ of sox9a primers (F_sox9a_E2 and R_sox9a_E3) and $150 \mathrm{nM} \beta$ actin primers (beta-actin_E3 and ${ }_{465}$ beta-actin_E4) in Fast SYBR ${ }^{\mathrm{TM}}$ Green Master Mix (4385612, ThermoFisher). $0.5 \mu \mathrm{L}$ of $\quad{ }_{466}$ template (1:10 dilution of reverse transcription reaction) was used in a $10 \mu \mathrm{L}$ reaction.

\section{Splinkerette-PCR-NGS}

13.1 Preparation of splinkerette library. To assess a wide spectrum of possible processed in parallel. $500 \mathrm{ng}$ of genomic DNA was digested with 20U of AluI (R0137, NEB) in a ${ }_{473}$ $30 \mu \mathrm{L}$ reaction at $37^{\circ} \mathrm{C}$ in a thermocycler with heated lid for 4 hours. The digest reaction was ${ }_{474}$ purified using phenol-chloroform extraction followed by ethanol precipitation and the pellet 475 resuspended with $34 \mu \mathrm{L}$ of nuclease-free water. Splinkerette adaptors (SPLINK-top_VC and 476 SPLINK-bottom_VC; Supp.Material) were annealed at $25 \mu \mathrm{M}$ final concentration each in $2.5 \mu \mathrm{L} \quad 477$ volume with the following cycling conditions: $95^{\circ} \mathrm{C} 2$ mins, decrease $95-22^{\circ} \mathrm{C}$ at $0.1{ }^{\circ} \mathrm{C} /$ second $\quad 478$ and ending with $22{ }^{\circ} \mathrm{C} 5$ mins before placing on ice. The ligation reaction was prepared by ${ }_{479}$ combining $17 \mu \mathrm{L}$ of purified AluI-digested genomic DNA with $0.5 \mu \mathrm{L}$ annealed adaptors and 480 10U of T4 DNA ligase (M0202, NEB) in 1X T4 DNA ligase buffer. Ligation was incubated ${ }_{481}$ 
overnight at $16{ }^{\circ} \mathrm{C}$ in a thermocycler with heated lid, subsequently purified using DNA Clean \& $\quad{ }_{482}$ Concentrator (D4003, Zymo Research) and eluted with $25 \mu \mathrm{L}$ DNA Elution Buffer. A primary ${ }^{483}$ PCR reaction $\left(12.5 \mu \mathrm{L}\right.$ volume) was setup by combining $10 \mathrm{ng}$ of ligated DNA with $0.25 \mu \mathrm{L}$ each $\quad{ }^{484}$ of $10 \mu \mathrm{M}$ SPLINK_P1 and DS-3' forward or DS-5' reverse P1 primers (Supp.Material) in 1X ${ }_{485}$ Platinum SuperFi master mix (12358010, ThermoFisher). Cycling was performed as follows: ${ }_{486}$ $98^{\circ} \mathrm{C} 2$ mins, $25 \mathrm{X}\left(98^{\circ} \mathrm{C} 10 \mathrm{~s}, 63^{\circ} \mathrm{C} 30 \mathrm{~s}, 72^{\circ} \mathrm{C} 3\right.$ mins $), 72^{\circ} \mathrm{C} 5$ mins, $12{ }^{\circ} \mathrm{C}$ hold. After cycling, ${ }^{487}$ $1 \mu \mathrm{L}$ of the primary PCR reaction was combined with $1 \mu \mathrm{L}$ each of $10 \mu \mathrm{M}$ SPLINK_P2 and DS-3' ${ }^{488}$ forward or DS-5' reverse P2 primers (Supp.Material) in 1X Platinum SuperFi master mix ${ }_{489}$ (50 $\mathrm{\mu L}$ volume) for secondary PCR. Cycling was performed like primary PCR, with the 490 exception that annealing temperature was lowered to $60^{\circ} \mathrm{C} .5 \mu \mathrm{L}$ of the secondary PCR 491 reaction was run on a $2 \%$ agarose gel to assess amplification of genomic regions flanking 492 integrations. Prior to library prep for Next Generation Sequencing (NGS), the secondary PCR ${ }_{493}$ reactions were purified using Select-a-Size DNA Clean \& Concentrator (D4080, Zymo Research) ${ }_{494}$ according to manufacturer's instructions to enrich for amplicons between 100-1000bp. Purified 495 amplicons were quantified by Qubit ${ }^{\mathrm{TM}}$ dsDNA HS Assay kit (Q32854, ThermoFisher) and ${ }_{496}$ $50 \mathrm{ng}$ used as starting input for library prep. Library prep was performed using Nextera DNA ${ }_{497}$ Library Prep Kit (FC-121-1030, Illumina) according to manufacturer's instructions and their ${ }_{498}$ fragment profiles ( 200bp expected) assessed on the TapeStation (Agilent Technologies). ${ }_{499}$ Libraries were sequenced on NextSeq500 Illumina platform (v2 150 cycles, 80-bp paired end) to 500 obtain $\sim 4$ million reads per forward/reverse sample.

13.2 Bioinformatics analysis. Obtained reads were quality trimmed using sickle (Joshi and 502 Fass, 2011) and -1 30 -q 30 parameters. Trimmed reads were mapped to the D.rerio genome 503 (GRCz10) using STAR (Dobin et al., 2013) with default parameters. Book-ended mapped 504 regions on the same strand were merged using bedtools merge (Quinlan and Hall, 2010)) to 505 'recapitulate' amplicon fragments prior to tagmentation during library prep. To identify regions 506 with significant signal over background in mapped reads (10 to 50-fold enrichment, ergo 507 integration sites), peaks were called using MACS2 callpeaks (Zhang et al., 2008) with -g 508 $1.41 \mathrm{e} 9$-m 1050 --nomodel --shiftsize X -q 0.01 parameters, where X=library 509 
fragment size/2. To filter out 'high-confidence' called peaks, bedtools intersect was used to 510 retrieve common peaks found in both forward and reverse samples overlapping by at least $1 \mathrm{bp}$. 511 If desired, peaks that overlapped annotated repeat elements by at least 1 bp were removed using 512 bedtools subtract. Genome ontology analysis of peaks were performed using HOMER 513 annotatePeaks.pl (Heinz et al., 2010) with -genomeOntology 514 intersect_positions_genomeOntology -gsize $1.41 \mathrm{e} 9$ parameters. 515

\section{Whole-mount immunohistochemistry}

Embryos were fixed in $4 \%$ paraformaldehyde (PFA) for 45 minutes at room temperature (RT). 517 After washing, embryos were blocked using 10\% goat serum in PBT (PBS, $0.5 \%$ Triton, 2\% ${ }_{518}$ DMSO) for 1 hour at RT. Primary antibodies used were chicken anti-GFP (to detect eGFP or 519 Citrine) (ab13970, Abcam) and rabbit anti-zfSox10 (GTX128374, GeneTex) in a 1:200 dilution, 520 added overnight at $4{ }^{\circ} \mathrm{C}$. Secondary antibodies used were donkey anti-rabbit 568nm (A10042, ${ }_{521}$ ThermoFisher) and goat anti-chicken 647nm (A21449,ThermoFisher) in 1:400 dilution each, $\quad{ }_{522}$ together with Hoechst reagent (H3569, ThermoFisher) in a 1:1000 dilution to label nuclei - all ${ }_{523}$ added for 2 hours at RT. After washing to remove excess, embryos were imaged using a LSM780 524 confocal microscope (Zeiss).

\section{Hybridisation Chain Reaction}

Embryos were fixed in $4 \%$ paraformaldehyde (PFA) overnight at $4{ }^{\circ} \mathrm{C}$, washed in ${ }_{527}$ phosphate-buffered saline (PBS), dehydrated in methanol $(\mathrm{MeOH})$ and stored at $-20^{\circ} \mathrm{C} . \quad{ }_{528}$ Hybridization chain reaction (HCR) v3.0 was performed according to published protocol (Choi ${ }_{529}$ et al., 2018). Briefly, embryos were rehydrated with a series of graded MeOH/PBS-Triton 530 (PBST) washes and incubated overnight at $37^{\circ} \mathrm{C}$ in $30 \%$ probe hybridization buffer containing 531 2 pmol of each probe mixture (pax3a and GFP). Excess probes were washed off with $30 \%$ probe 532 wash buffer at $37^{\circ} \mathrm{C}$ and $5 \mathrm{XSSCT}$ at RT. Embryos were then incubated overnight at RT in ${ }_{533}$ amplification buffer containing 15 pmol of each fluorophore-labelled hairpin (B3-546 and 534 B2-488). Excess hairpins were removed by washing with 5XSSCT at RT. Following HCR, 535 embryos were imaged using a LSM780 confocal microscope (Zeiss). 


\section{Competing interests}

\section{Author contributions}

\section{Acknowledgements and funding}

This work was supported by MRC (G0902418), Lister Institute Research Prize, Oxford BHF 545 CRE award (RE/08/004) to T.S.-S. and (RE/13/1/30181) to F.C.S., RDM Pump Priming ${ }_{546}$ Grant (HSD00040) to T.S.-S. and V.C.-M., Clarendon Fund Fellowship to V.C.-M, BHF Programme grant and Chair award (RG/13/9/303269 and CH/11/1/28798) to P.R.R.. We ${ }_{548}$ would like to thank Wenbiao Chen for advice on choice of zebrafish U6a promoter, Sarah de Val ${ }_{549}$ for E1b minimal promoter cassette, Joey Riepsaame for dCas9-SID4x construct, and current 550 collaborators who have requested the enhancer construct for their work.

\section{References} expression in the 3D genome. Nature Reviews Molecular Cell Biology, 17(12):771-782, 2016.554 ISSN 14710080. doi: 10.1038/nrm.2016.138. amplifiers that control nuclear structure and gene expression. Nature Reviews Molecular Cell 557 Biology, 17(12):756-770, 2016. ISSN 1471-0072. doi: 10.1038/nrm.2016.126. 
Ryan Rickels and Ali Shilatifard. Enhancer Logic and Mechanics in Development and Disease. 559 Trends in Cell Biology, 28(8):608-630, 2018. ISSN 18793088. doi: 10.1016/j.tcb.2018.04.003. 560

Jeffrey J. Quinn and Howard Y. Chang. Unique features of long non-coding RNA biogenesis ${ }_{561}$ and function. Nature reviews. Genetics, 17(1):47-62, 2015. ISSN 1471-0064. doi: 562 10.1038/nrg.2015.10.

Andrew R Bassett, Asifa Akhtar, Denise P Barlow, Adrian P Bird, Neil Brockdorff, Denis 564 Duboule, Anne Ephrussi, Anne C Ferguson-Smith, Thomas R Gingeras, Wilfried Haerty, 565 Douglas R Higgs, Eric a Miska, and Chris P Ponting. Considerations when investigating 566 lncRNA function in vivo. eLife, 3:1-14, 8 2014. ISSN 2050-084X. doi: 10.7554/eLife.03058. $\quad 567$

Barbara McClintock. The Origin and Behavior of Mutable Loci in Maize. Genetics, 36(6): ${ }_{568}$ 344-355, 1950. ISSN 0027-8424. doi: 10.1073/pnas.36.6.344.

N. Fedoroff, S. Wessler, and M. Shure. Isolation of the transposable maize controlling elements 570 Ac and Ds. Cell, 35(1):235-242, 1983. ISSN 00928674. doi: 10.1016/0092-8674(83)90226-X. $\quad 571$

Alexander Emelyanov, Yuan Gao, Naweed Isaak Naqvi, and Serguei Parinov. Trans-kingdom 572 transposition of the maize Dissociation element. Genetics, 174(3):1095-1104, 2006. ISSN 573 00166731. doi: 10.1534/genetics.106.061184.

Alexander Emelyanov and Serguei Parinov. Mifepristone-inducible LexPR system to drive and 575 control gene expression in transgenic zebrafish. Developmental Biology, 320(1):113-121, 2008. 576 ISSN 00121606. doi: 10.1016/j.ydbio.2008.04.042.

A. Kenyon, D. Gavriouchkina, J. Zorman, V. Chong-Morrison, G. Napolitani, V. Cerundolo, ${ }_{578}$ and T. Sauka-Spengler. Generation of a double binary transgenic zebrafish model to study 579 myeloid gene regulation in response to oncogene activation in melanocytes. DMM Disease $\quad 580$ Models and Mechanisms, 11(4), 2018. ISSN 17548411. doi: 10.1242/dmm.030056. 581

Helen Ngoc Bao Quach, Shijie Tao, Pavle Vrljicak, Adita Joshi, Hua Ruan, Rashmi Sukumaran, 582 Gaurav K Varshney, Matthew C LaFave, Shawn M Burgess, Christoph Winkler, Alexander ${ }_{583}$ Emelyanov, Sergey Parinov, and Karuna Sampath. A Multifunctional Mutagenesis System 584 
for Analysis of Gene Function in Zebrafish. G3 (Bethesda, Md.), 5(6):1283-99, 2015. ISSN 585 2160-1836. doi: 10.1534/g3.114.015842.

Koichi Kawakami. Tol2: a versatile gene transfer vector in vertebrates. Genome Biology, $8 \quad 587$ (Suppl 1):S7, 2007. ISSN 14656906. doi: 10.1186/gb-2007-8-s1-s7.

Pavle Vrljicak, Shijie Tao, Gaurav K. Varshney, Helen Ngoc Bao Quach, Adita Joshi, $\quad 589$

Matthew C. LaFave, Shawn M. Burgess, and Karuna Sampath. Genome-Wide Analysis of 590 Transposon and Retroviral Insertions Reveals Preferential Integrations in Regions of DNA ${ }_{591}$ Flexibility. G3: Genes, Genomes, Genetics, 6(4):805-817, 2016. ISSN 2160-1836. doi: 592 $10.1534 / \mathrm{g} 3.115 .026849$.

Lei S Qi, Matthew H Larson, Luke a Gilbert, Jennifer a Doudna, Jonathan S Weissman, ${ }_{594}$ Adam P Arkin, and Wendell a Lim. Repurposing CRISPR as an RNA-guided platform for 595 sequence-specific control of gene expression. Cell, 152(5):1173-83, 2 2013. ISSN 1097-4172. 596 doi: 10.1016/j.cell.2013.02.022.

Luke A Gilbert, Matthew H Larson, Leonardo Morsut, Zairan Liu, Gloria A Brar, Sandra E 598 Torres, Noam Stern-Ginossar, Onn Brandman, Evan H Whitehead, Jennifer A Doudna, 599 Wendell A Lim, Jonathan S Weissman, and Lei S Qi. CRISPR-mediated modular 600 RNA-guided regulation of transcription in eukaryotes. Cell, 154(2):442-51, 7 2013. ISSN 601 1097-4172. doi: 10.1016/j.cell.2013.06.044. 
Silvana Konermann, Mark D. Brigham, Alexandro E. Trevino, Julia Joung, Omar O. 612 Abudayyeh, Clea Barcena, Patrick D. Hsu, Naomi Habib, Jonathan S. Gootenberg, Hiroshi ${ }_{613}$ Nishimasu, Osamu Nureki, and Feng Zhang. Genome-scale transcriptional activation by an $\quad{ }^{614}$ engineered CRISPR-Cas9 complex. Nature, 517(7536):583-8, 2015. ISSN 0028-0836. doi: ${ }_{615}$ $10.1038 /$ nature14136.

S J Liu, S John Liu, Max A Horlbeck, Seung Woo Cho, Harjus S Birk, Martina Malatesta, ${ }_{617}$ Frank J Attenello, Jacqueline E Villalta, Min Y Cho, Yuwen Chen, A Mandegar, Michael P ${ }_{618}$ Olvera, Luke A Gilbert, Bruce R Conklin, Howard Y Chang, Jonathan S Weissman, and ${ }_{619}$ Daniel A Lim. CRISPRi-based genome-scale identification of functional long noncoding RNA 620 loci in human cells. 7111(December), 2016. ISSN 0036-8075. doi: 10.1126/science.aah7111. ${ }_{621}$

Ruth M Williams, Upeka Senanayake, Mara Artibani, Gunes Taylor, Daniel Wells, 622 Ahmed Ashour Ahmed, and Tatjana Sauka-Spengler. Genome and epigenome engineering ${ }_{623}$ CRISPR toolkit for in vivo modulation of cis -regulatory interactions and gene expression in $\quad{ }^{624}$ the chicken embryo. Development, page dev.160333, 2018. ISSN 0950-1991. doi: 625 10.1242/dev.160333.

Prashant Mali, John Aach, P Benjamin Stranges, Kevin M Esvelt, Mark Moosburner, Sriram ${ }_{627}$ Kosuri, Luhan Yang, and George M Church. CAS9 transcriptional activators for target ${ }_{628}$ specificity screening and paired nickases for cooperative genome engineering. Nature ${ }^{629}$ biotechnology, 31(9):833-8, 9 2013. ISSN 1546-1696. doi: 10.1038/nbt.2675.

Aamir Mir, Julia F. Alterman, Matthew R. Hassler, Alexandre J. Debacker, Edward Hudgens, ${ }_{631}$ Dimas Echeverria, Michael H. Brodsky, Anastasia Khvorova, Jonathan K. Watts, and Erik J. 632 Sontheimer. Heavily and fully modified RNAs guide efficient SpyCas9-mediated genome ${ }_{633}$ editing. Nature Communications, 9(1):1-9, 2018. ISSN 20411723. doi: 634 10.1038/s41467-018-05073-Z.

Philipp W. Becker, Natalia Sacilotto, Svanhild Nornes, Alice Neal, Max O. Thomas, Ke Liu, ${ }_{636}^{63}$ Chris Preece, Indrika Ratnayaka, Benjamin Davies, George Bou-Gharios, and Sarah De Val. ${ }_{637}$ An intronic Flk1 enhancer directs arterial-specific expression via RBPJ-mediated venous ${ }_{638}$ 
repression. Arteriosclerosis, Thrombosis, and Vascular Biology, 36(6):1209-1219, 2016. ISSN 639 15244636. doi: 10.1161/ATVBAHA.116.307517.

Koichi Kawakami. Transgenesis and Gene Trap Methods in Zebrafish by Using the Tol2

Transposable Element. Methods in Cell Biology, 77:201-222, 2004. ISSN 0091679X. doi: ${ }_{642}$ 10.1016/S0091-679X(04)77011-9.

Le A. Trinh, Vanessa Chong-Morrison, Daria Gavriouchkina, Tatiana Hochgreb-Hägele, Upeka ${ }_{644}$ Senanayake, Scott E. Fraser, and Tatjana Sauka-Spengler. Biotagging of Specific Cell ${ }_{645}$ Populations in Zebrafish Reveals Gene Regulatory Logic Encoded in the Nuclear ${ }_{646}$ Transcriptome. Cell Reports, 19(2):425-440, 2017. ISSN 22111247. doi: 647 10.1016/j.celrep.2017.03.045.

Daria Gavriouchkina, Ruth M Williams, Martyna Lukoseviciute, Tatiana Hochgreb-Hägele,

Tatiana Hochgreb-Hägele and Marianne E Bronner. A novel FoxD3 gene trap line reveals 653 neural crest precursor movement and a role for FoxD3 in their specification. Developmental ${ }_{654}$ biology, 374(1):1-11, 2 2013. ISSN 1095-564X. doi: 10.1016/j.ydbio.2012.11.035. 
Jason D Buenrostro, Paul G Giresi, Lisa C Zaba, Howard Y Chang, and William J Greenleaf. ${ }_{665}$ Transposition of native chromatin for fast and sensitive epigenomic profiling of open 666 chromatin, DNA-binding proteins and nucleosome position. Nature methods, 10(12):1213-8, ${ }_{667}$ 2013. ISSN 1548-7105. doi: 10.1038/nmeth.2688.

Harry M T Choi, Maayan Schwarzkopf, Mark E Fornace, Aneesh Acharya, Georgios Artavanis, ${ }_{669}$ Johannes Stegmaier, Alexandre Cunha, and Niles A Pierce. Third-generation in situ 670 hybridization chain reaction: multiplexed, quantitative, sensitive, versatile, robust. 671 Development, 145(12):dev165753, 2018. ISSN 0950-1991. doi: 10.1242/dev.165753. 672

Linlin Yin, Lisette A. Maddison, Mingyu Li, Nergis Kara, Matthew C. Lafave, Gaurav K. $\quad 673$ Varshney, Shawn M. Burgess, James G. Patton, and Wenbiao Chen. Multiplex conditional 674 mutagenesis using transgenic expression of Cas9 and sgRNAs. Genetics, 200(2):431-441, $\quad 675$ 2015. ISSN 19432631. doi: 10.1534/genetics.115.176917.

Brian D. Clarke, David M. Cummins, Ken a. McColl, Alister C. Ward, and Tim J. Doran. 677 Characterization of Zebrafish Polymerase III Promoters for the Expression of Short-Hairpin 678 RNA Interference Molecules. Zebrafish, 10(4):121002061007008, 2012. ISSN 1545-8547. doi: 679 10.1089/zeb.2012.0782.

Mihaela Pertea, Geo M Pertea, Corina M Antonescu, Tsung-Cheng Chang, Joshua T Mendell, ${ }_{681}$ and Steven L Salzberg. StringTie enables improved reconstruction of a transcriptome from ${ }_{682}$ RNA-seq reads. Nature Biotechnology, 33(3):290-295, 2015. ISSN 1087-0156. doi: 683 $10.1038 /$ nbt. 3122 . 
gene structure, evolution, and expression. Genome research, 22(9):1775-89, 9 2012. ISSN 691 1549-5469. doi: 10.1101/gr.132159.111.

Mehdi Goudarzi, Kathryn Berg, Lindsey M Pieper, and Alexander F Schier. Long non-coding 693 RNAs are largely dispensable for zebrafish embryogenesis, viability and fertility. bioRxiv, ${ }_{694}$ pages 1-22, 2018. doi: 10.1101/374702.

Jesse G. Zalatan, Michael E. Lee, Ricardo Almeida, Luke A. Gilbert, Evan H. Whitehead, ${ }_{696}$ Marie La Russa, Jordan C. Tsai, Jonathan S. Weissman, John E. Dueber, Lei S. Qi, and ${ }_{697}$ Wendell A. Lim. Engineering complex synthetic transcriptional programs with CRISPR RNA ${ }_{698}$ scaffolds. Cell, 160(1-2):339-350, 2015. ISSN 10974172. doi: 10.1016/j.cell.2014.11.052. ${ }^{699}$

Deborah Hay, Jim R. Hughes, Christian Babbs, James O.J. Davies, Bryony J. Graham, 700 Lars L.P. Hanssen, Mira T. Kassouf, A. Marieke Oudelaar, Jacqueline A. Sharpe, Maria C. 701 Suciu, Jelena Telenius, Ruth Williams, Christina Rode, Pik Shan Li, Len A. Pennacchio, 702 Jacqueline A. Sloane-Stanley, Helena Ayyub, Sue Butler, Tatjana Sauka-Spengler, Richard J. 703 Gibbons, Andrew J.H. Smith, William G. Wood, and Douglas R. Higgs. Genetic dissection of 704 the $\alpha$-globin super-enhancer in vivo. Nature Genetics, 48(8):895-903, 2016. ISSN 15461718. 705 doi: $10.1038 /$ ng.3605.

Anja J. Will, Giulia Cova, Marco Osterwalder, Wing Lee Chan, Lars Wittler, Norbert Brieske, 707 Verena Heinrich, Jean Pierre De Villartay, Martin Vingron, Eva Klopocki, Axel Visel, 708 Darío G. Lupianez, and Stefan Mundlos. Composition and dosage of a multipartite enhancer 709 cluster control developmental expression of Ihh (Indian hedgehog). Nature Genetics, 49(10): 710 1539-1545, 2017. ISSN 15461718. doi: 10.1038/ng.3939.

Daniel G Gibson, Lei Young, Ray-Yuan Chuang, J Craig Venter, Clyde a Hutchison, 712 Hamilton O Smith, Clyde A Hutchison Iii, and Nature America. Enzymatic assembly of DNA 713 molecules up to several hundred kilobases. Nature Methods, 6(5):343-345, 2009. ISSN 714 1548-7105. doi: 10.1038/nmeth.1318. 
Transposon-mediated BAC transgenesis in zebrafish. Nature Protocols, 6(12):1998-2021, 2011. 717 ISSN 1750-2799. doi: 10.1038/nprot.2011.416.

NA Joshi and JN Fass. Sickle: A sliding-window, adaptive, quality-based trimming tool for 719 FastQ files (Version 1.33), 2011. URL https://github.com/najoshi/sickle. 720

Alexander Dobin, Carrie A. Davis, Felix Schlesinger, Jorg Drenkow, Chris Zaleski, Sonali Jha, ${ }^{721}$ Philippe Batut, Mark Chaisson, and Thomas R. Gingeras. STAR: Ultrafast universal 722 RNA-seq aligner. Bioinformatics, 29(1):15-21, 2013. ISSN 13674803. doi: 723 10.1093/bioinformatics/bts635. genomic features. Bioinformatics, 26(6):841-842, 2010. ISSN 13674803. doi: 726 10.1093/bioinformatics/btq033.

Yong Zhang, Tao Liu, Clifford A. Meyer, Jérôme Eeckhoute, David S. Johnson, Bradley E. ${ }_{728}$ Bernstein, Chad Nussbaum, Richard M. Myers, Myles Brown, Wei Li, and X. Shirley Shirley. ${ }^{729}$ Model-based analysis of ChIP-Seq (MACS). Genome Biology, 9(9), 2008. ISSN 14747596.730 doi: 10.1186/gb-2008-9-9-r137.

Sven Heinz, Christopher Benner, Nathanael Spann, Eric Bertolino, Yin C. Lin, Peter Laslo, ${ }_{732}$ Jason X. Cheng, Cornelis Murre, Harinder Singh, and Christopher K. Glass. Simple 733 Combinations of Lineage-Determining Transcription Factors Prime cis-Regulatory Elements 734 Required for Macrophage and B Cell Identities. Molecular Cell, 38(4):576-589, 2010. ISSN 735 10972765. doi: 10.1016/j.molcel.2010.05.004. 